provided the methodology for studying of the lived experience of 15 participants aged 18 and 25 years living in Ireland who had a self-reported chronic illness since childhood. Drawing on the philosophy of van Manen's lifeworld existentials, the lived experiences were revealed. Emerging adults struggle to achieve a sense of identity which is often sabotaged by illness. The findings of this study conclude that a sense of connectedness with peers was important for emerging adults with illness as they journeyed towards developing a sense of identity.

\title{
OC18 - Neurodevelopmental outcomes following congenital heart surgery
}

Eleni Syrgani (Greece) $)^{1,2}$; Konstantinos Petsios (Greece) $)^{1,2}$

${ }^{1}$ National Kapodistrian University of Athens; ${ }^{2}$ 'Onassis' Cardiac Center-PICU

Theme: Complex health care and chronic disease management.

Keywords: Congenital heart disease, developmental disabilities, outcome, neurodevelopment delay.

Introduction: Congenital heart disease (CHD) may have a very important impact on central nervous system function and neurodevelopment.

Purpose: Critical evaluation of literature concerning the neurodevelopmental outcomes following congenital heart surgery.

Methods: Systematic review of 28 articles published after 2000 in PubMed, Scopus and CINAHL.

Results: Neurodevelopment morbidities can have a negative impact on early childhood development, academic performance and later transition to adulthood. The spectrum of neurodevelopment impairment is wide, depending on the complexity of $\mathrm{CHD}$ and it is characterized by mild cognitive impairment, impaired social interaction, and impairments in core communication skills, including pragmatic language, as well as inattention, impulsive behavior and impaired executive function. A number of important determinants are presented.

Conclusions: Children with CHD are at increased risk for neurodevelopmental delay. Screening and evaluation of neurodevelopmental delay, along with regular follow up, are essential steps to guide appropriate interventions to optimize their overall development.

\section{OC19 - Measuring feasibility, reliability and validity of the Greek version of PedsQL cardiac module}

Maria Drakouli (Greece) ${ }^{1,2}$; Konstantinos Petsios (Greece) ${ }^{1,2}$;

Vasiliki Matziou (Greece) ${ }^{1}$

${ }^{1}$ National and Kapodistrian University of Athens; ${ }^{2}$ Onassis Cardiac Center-PICU 
Theme: Cardiology

Keywords: Adolescents, children, quality of life, PedsQL Cardiac Module, validation.

Introduction: Measuring quality of life (QoL) in children and adolescents with congenital heart disease $(\mathrm{CHD})$ is of great clinical importance.

Objective: The aim of the study was: (a) to adapt the PedsQL Cardiac Module for children aged two to 18 years with $\mathrm{CHD}$ in a sample of the Greek population; (b) to determine its reliability and validity.

Methods: Forward and backward translation methodology was used. Parents and children completed the instrument during: (a) hospitalization and (b) visits in the paediatric cardiology outpatient department. Cross-informant variance between children and parents was thoroughly assessed.

Results: Missing item responses did not exceed 5\%. All internal consistency reliability coefficients for the inventory exceeded the minimum standards for group comparisons, over 0.75 . Hypothesized correlations between cardiac module and core scales were statistically significant, $(p<0.05)$. Agreement between children and parents was relatively high. Pilot study results will be additionally presented.

Conclusion: The findings support the feasibility, reliability and validity of the Greek translation of the PedsQL Cardiac Module in children with congenital heart defect (CHD).

\title{
OC20 - Skin-to-skin contact in the first hour of life
}

\author{
Manuela Ferreira (Portugal)i; Tânia Vaz (Portugal)2; \\ Graça Aparício (Portugal)ํㅜ; João Duarte (Portugal) ${ }^{1}$
}

\section{${ }^{1}$ Instituto Politécnico de Viseu - Escola Superior de Saúde CI\&DETS; ${ }^{2}$ Centro Hospitalar Cova da Beira}

Theme: Child protection and managing risk.

Keywords: Breastfeeding, newborn, skin-to-skin contact.

Background: Skin-to-skin contact in the first hour of life has benefits for the mother and the newborn, as well as a major role in establishing breastfeeding.

Objectives: Determine the prevalence of skin-to-skin contact and breastfeeding within the first hour of life.

Method: A systematic review of literature followed by a quantitative and simple descriptive cross-sectional study, according to a non-probability analyzing of 382 clinic records of postpartum mothers.

Results: Evidence that early skin-to-skin contact immediately after birth is a potential sensory stimulus, which covers the newborn warming, tactile and active stimulation, respiratory rates and level of blood glucose, reduces baby crying and promotes breastfeeding. In the sample, about $92.6 \%$ of the mothers put the baby to the breast in the first hour of life, but only $26.6 \%$ made skin-to-skin contact with the baby.

Conclusion: Despite the scientific evidence of the benefits of skin-to-skin contact 